\title{
LED-Based Light Source Combined with Quantum Dot for Spectral Imaging
}

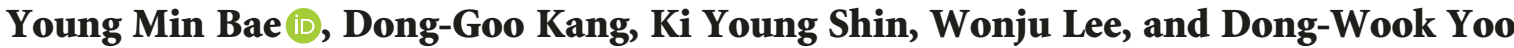

Korea Electrotechnology Research Institute, 111 Hanggaul-ro, Ansan 15588, Republic of Korea

Correspondence should be addressed to Young Min Bae; kimbym@keri.re.kr

Received 23 January 2019; Revised 12 March 2019; Accepted 9 April 2019; Published 20 May 2019

Guest Editor: Qinghua Li

Copyright ( 2019 Young Min Bae et al. This is an open access article distributed under the Creative Commons Attribution License, which permits unrestricted use, distribution, and reproduction in any medium, provided the original work is properly cited.

\begin{abstract}
Quantum dot (QD) is a kind of semiconductor nanoparticle and shows photoluminescence, in which the light with narrow spectral band is emitted from the QD after the absorption of the excitation light. In this study, it is demonstrated that the LED-based light source combined with a QD film can be applied to spectral imaging. The polymer film with QD was prepared by photopolymerization. Through measurement of the emission spectrum, it was confirmed that the optical intensity at the peak wavelength of the spectral bands of 410 and $540 \mathrm{~nm}$ could be controlled by changing the thickness of the film. In the study using a home-made phantom mimicking blood vessels, the patterns' contrast in the phantom images could be enhanced by using LED combined with QD as compared with the white light image. The LED light combined with the QD film provides a useful solution for illumination for spectral imaging.
\end{abstract}

\section{Introduction}

Quantum dot (QD) is a kind of semiconductor nanoparticle and has photoluminescence, in which the light with narrow spectral band is emitted from the QD after excitation light is absorbed [1]. QD as a fluorophore with high quantum efficiency has been applied to quantum dot-emitting diode, display, and biomedical technologies [1-5]. In particular, cellular labeling and imaging and assay labeling with QD bioconjugate were very popular in the biomedical field, and their solutions based on QD were commercialized $[4,5]$. In this study, it is demonstrated that the QD can be applied to a light source for spectral imaging as a new approach for the application of QD.

Optical imaging has been applied in medical diagnostics such as endoscopy to provide an optical image of disease sites or physiological conditions in real time. The optical endoscope basically provides white light images which show the surface structures of blood vessel patterns or polyps on tissue [6]. Although white light imaging has been successfully used to diagnose the disease, novel optical imaging modalities such as spectral imaging or fluorescence imaging emerged to visualize morphological changes of microvessel or tumors with high contrast [7-9]. For example, narrow band imaging (NBI) and blue laser imaging (BLI), which are kinds of spectral imaging, are provided by the commercial manufacturers of endoscopes [10, 11]. In NBI, which is well known to be clinically helpful, the light with the spectral bands near 410 and $540 \mathrm{~nm}$ illuminates tissues through the light guide [12]. In fluorescence imaging, the light of the excitation band of endogenous or exogenous fluorescent substances illuminates tissues in order to induce the emission of the light shifted to the higher wavelength [13]. In modern endoscopes, as the diameter of the insertion tube which is inserted inside the human body is very small (at least $\sim 10 \mathrm{~mm}$ ), the light for illumination is delivered to the end of the insertion tube through a light guide from a light source [14]. Also, illumination of the light of spectrally narrow band is commonly needed for the spectral imaging or the fluorescence imaging. Several types of light source have been implemented to generate the light of spectrally narrow band. The NBI which was implemented in Olympus Corp. uses a xenon lamp with the filter wheel in which multiple band-pass filters are mounted [12]. As the xenon lamp provides the broad spectrum of light in the range from visible to near-infrared wavelength, the light in the specific spectral band can be outputted with the optical 
band-pass filter. However, as the xenon lamp produces heat too much, the bulky structure for dissipating heat is needed, and the high maintenance expense is required due to the short lifetime. Recently, applications of the solid state light sources in endoscopy have been reported to substitute the xenon lamp [15]. In particular, the light source with the broad band spectrum induced from phosphor by the blue laser was applied to implement spectral imaging in the clinical study [11]. Also, the combination of multiple color lightemitting diodes (LEDs) of blue, green, and red was implemented to generate the light of the multispectral band or white light $[15,16]$. Although LEDs provide the advantages such as high efficiency, low cost, and long lifetime, this approach based on the use of multiple LEDs needs multiple driver modules to supply power to each LED and has a limitation on the expansion to spectral imaging with the lights of more bands.

In this study, it is reported that the LED combined with a QD film can be used as the light source with multiple spectral bands. The semitransparent film with QD is fabricated, and the light of the multispectral band is implemented by combining the QD film with a single LED. Then, it is demonstrated that the light source can be applied to spectral imaging through the experimental study with a home-made phantom. As a result, the QD as a nanomaterial can provide the new solution for the illumination of spectral imaging.

\section{Materials and Method}

2.1. Fabrication of the QD Film. Two QD materials (green QD (G6 type) and red QD (G6 type)) were purchased from QDBrick Corp (Korea). The QDs with a core-shell structure of CdSe/ZnSe were a form of powder and were used without any treatment [17]. QD films were prepared by a photopolymerization process [18]. The QD powder was mixed with photopolymerizable epoxy monomer (NOA63, Thorlabs Inc.) followed by degassing under vacuum. Then, the mixture was poured in a PDMS mold with engraved disk pattern and was exposed to UV light of about $100 \mathrm{~mW} / \mathrm{cm}^{2}$ for 60 seconds. The QD film was completed by peeling the PDMS mold.

2.2. Experimental Setup. The images of phantom were acquired with the optical configuration shown in Figure 1. The QD film was excited with the ultraviolet (UV) LED (CBM-120-UV, Luminus Inc.), and the emitted light from a QD film was transmitted to a phantom through a collimation lens. A monochromatic camera (XiQ, Ximea Corp.) was used to acquire the images of phantom. Phantom's white light image was acquired using the configuration in which a white light LED (CBT-140, Luminus Inc.) was used instead of the UV LED and the QD film was removed in Figure 1. No optical filters were used in all the acquisition of images.

\section{Results and Discussion}

Spectral imaging needs the sequential or simultaneous illumination with light of several spectral bands. For example, lights with a spectral band of 410 and $540 \mathrm{~nm}$, the main

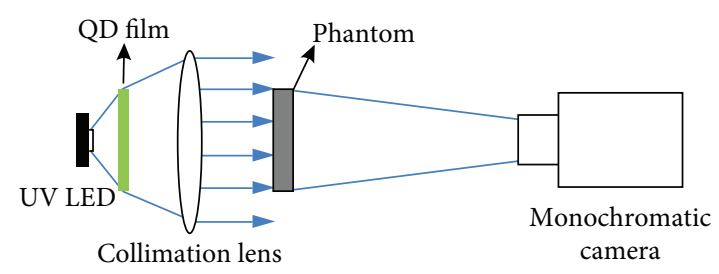

FIGURE 1: Experimental setup for imaging under illumination of LED combined with QD film.

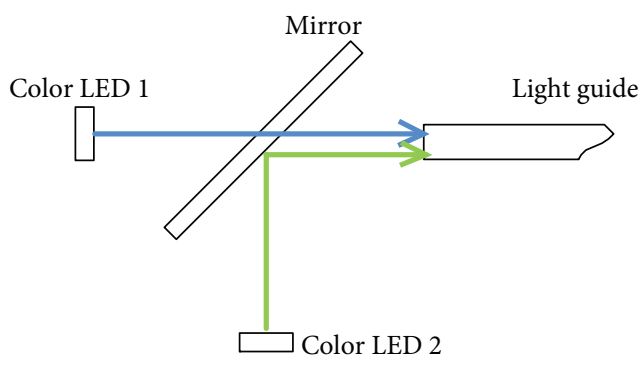

(a)

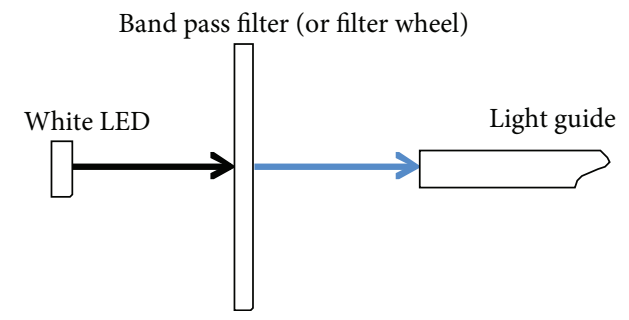

(b)

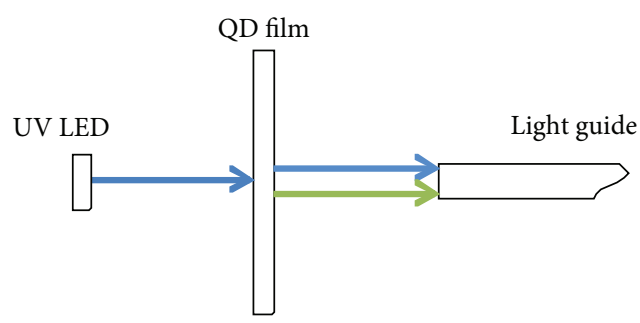

(c)

FIGURE 2: Configuration for illumination with light of spectral bands.

absorption band of hemoglobin, are used to enhance blood vessel patterns in medical spectral imaging [19]. Conventional methods for generating the spectral band light with LEDs combine multiple light sources with optical components, such as lenses and mirrors, as shown in Figure 2(a) [20]. In this case, multiple drivers for each light source and appropriate optical design for combining lights should be considered in the configuration. Figure 2(b) shows an approach to generate light with band-pass filters, in which a single white light LED with broadband emission spectrum and band-pass filters is used [21]. Properly selected filters can produce light in the spectral band. However, since the conventional white light LED does not cover the spectral band around $410 \mathrm{~nm}$, the configuration shown in Figure 2(b) has limitations in spectral imaging to improve the contrast of 

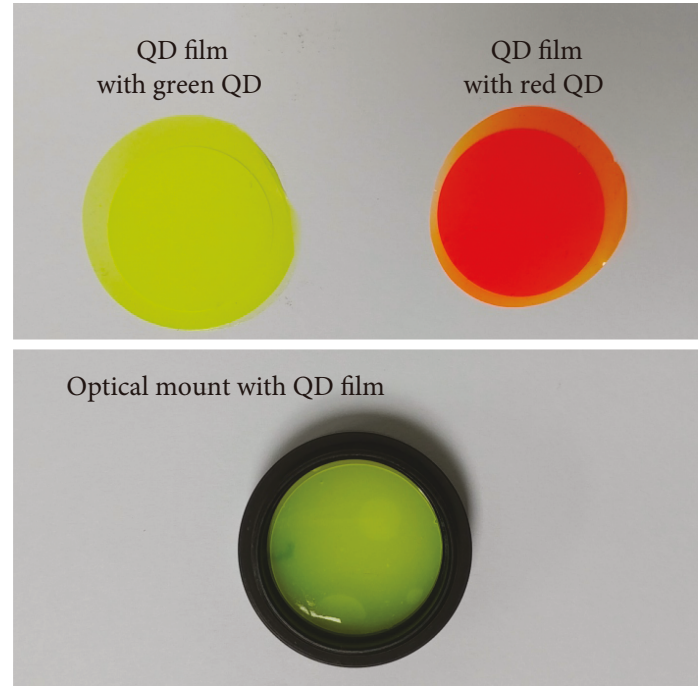

(a)

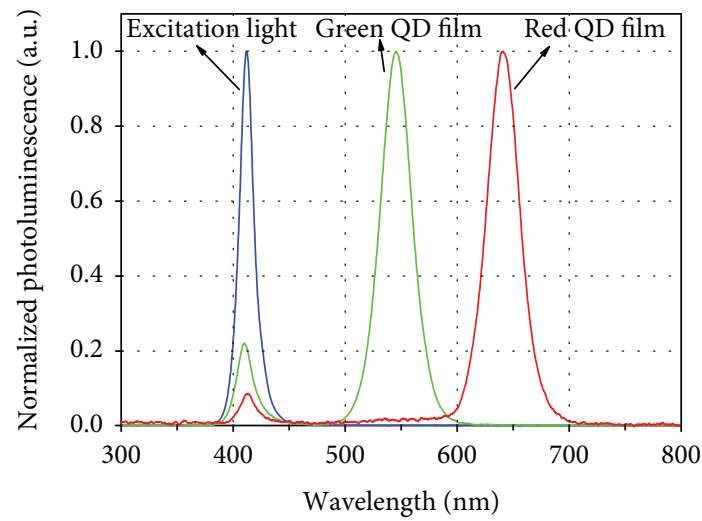

(b)

FIGURE 3: QD films fabricated with the photopolymerization process. (a) Upper image showing the photographs of QD films with green QD and red QD and the lower image showing the oneinch optical mount with QD film. (b) Intensity spectrum of the excitation light and the emission spectrums of the QD film with green QD and red QD.

blood vessels. In this study, a multispectral band light was implemented with the configuration shown in Figure 2(c). This new type of illumination consists of a single LED and a QD film. The LED is used to excite the QD in the QD film, which is a polymer film with QD nanoparticle which has emission in desirable wavelength.

The upper image of Figure 3(a) shows the typical images of QD films which is $300 \mu \mathrm{m}$ thick. The QD powder and the epoxy monomer were mixed in the weight-based ratio of 93 : 7; then, the QD film was prepared by photopolymerizing the mixture as described above. The films are easily attached to a glass substrate, and the lower image of Figure 3(a) shows the QD film embedded in the one-inch optical mount with a glass plate. Figure 3(b) shows the intensity spectrum of the excitation light and the emission spectrums of the QD films. The center wavelength and FWHM (full width at half maximum) of excitation light were $411.8 \mathrm{~nm}$ and $15.0 \mathrm{~nm}$, respectively, and the center wavelength of light emitted

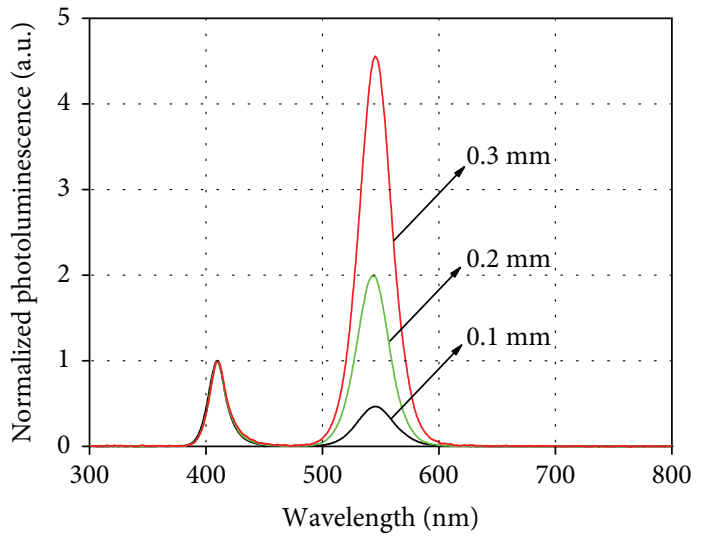

FIgURE 4: Change of the emission spectrums of the QD film with green QD according to the film thickness.

from the QD film of green QD and red QD was $541.3 \mathrm{~nm}$ and $641.0 \mathrm{~nm}$, respectively. The photoluminescence of the quantum dots in the narrow spectral band is attributed to the quantum confinement effect due to the small size of $\mathrm{QD}$, and the emission peak depends on the energy band gap determined by QD size $[1,22]$. Since QDs with various spectral emission bands have already been commercialized, it is possible to implement a light source with desired spectral bands in the visible wavelength range. The small peaks near $410 \mathrm{~nm}$ observed in each spectrum of the green QD and red QD films are due to the photons transmitted without being absorbed by the QD. In the QD films with green QD and red $\mathrm{QD}$, the FWHM at the maximum peak band was $32.3 \mathrm{~nm}$ and $34.0 \mathrm{~nm}$, respectively. As the bandwidth of the QD emission is due to the distribution of the QD size, the narrower band can be achieved by homogenizing the size of the QD. Considering that the bandwidth of the spectral illumination used in the NBI is about $30 \mathrm{~nm}$, the bandwidth of the light emitted by the QD film was estimated to be applicable to endoscopic spectral imaging $[10,23]$.

The emission spectrum of QD films with green QD changes depending on their thickness as shown in Figure 4. In the figure, the peak in the band near $410 \mathrm{~nm}$ is due to the excitation light, and the peak in the spectral band near $540 \mathrm{~nm}$ is the emission band of the green QD. And, the value of the $y$-axis means the ratio of the light intensity to that at the peak wavelength near the band of $410 \mathrm{~nm}$. The peak position in the band around $540 \mathrm{~nm}$ was almost unchanged with a standard deviation of $1.3 \mathrm{~nm}$ depending on the thickness. On the other hand, as the thickness increases, the light intensity decreases in the $410 \mathrm{~nm}$ band and the light intensity increases in the $540 \mathrm{~nm}$ band. This phenomenon can be explained by Beer's law, where the absorbance is proportional to the concentration of the substance and the penetration depth [24]. As the penetration depth corresponds to the film thickness, the ratio $\left(R_{540 / 410}\right)$ of the light intensity at the peak wavelength of $540 \mathrm{~nm}$ band to the light intensity at the peak wavelength of $410 \mathrm{~nm}$ band was $0.47,2.0$, and 4.6 at 100,200 , and $300 \mu \mathrm{m}$ thickness, respectively. Because the absorption of material along the wavelength depends on the molecular structure, the irradiation of the multispectral band 


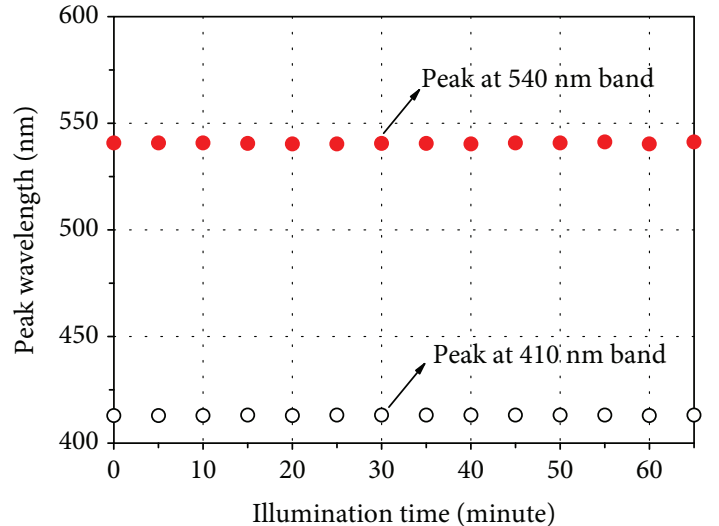

(a)

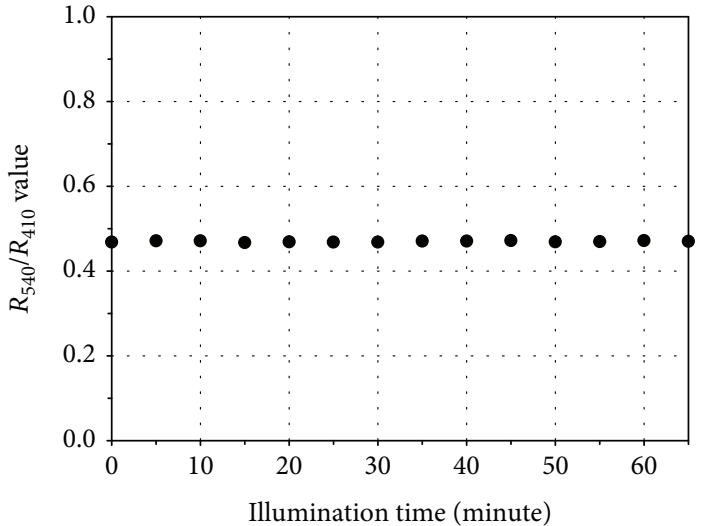

(b)

FIGURE 5: Changes of the emission of QD film during the continuous illumination time. (a) Change of the position of peak wavelength at both spectral bands of 410 and $540 \mathrm{~nm}$. (b) Change of $R_{540 / 410}$ value.

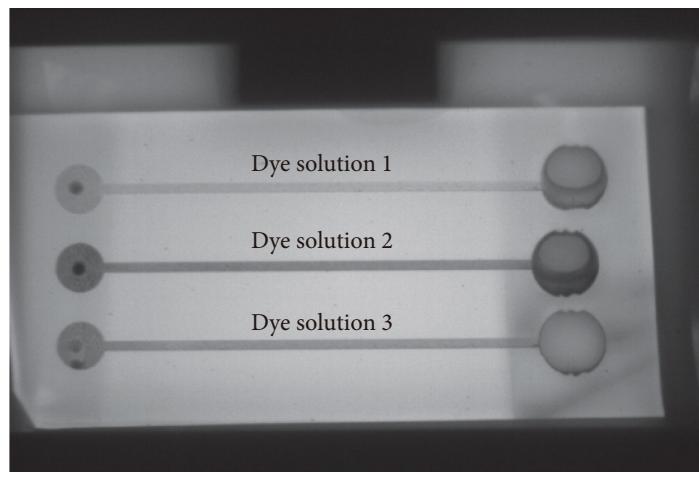

(a)

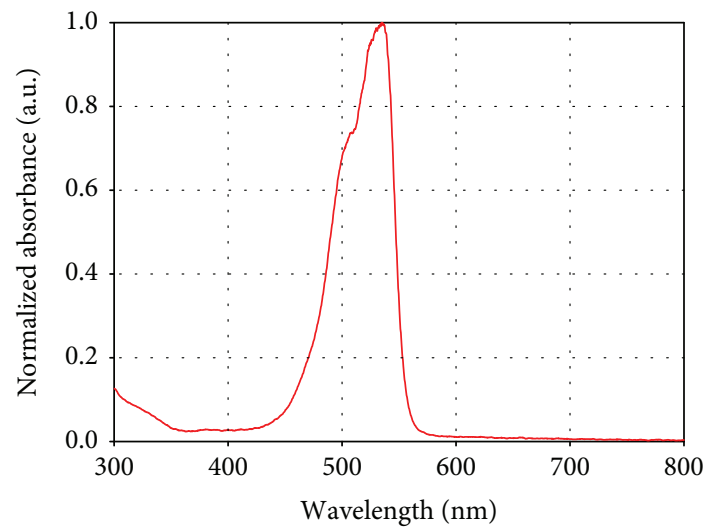

(c)

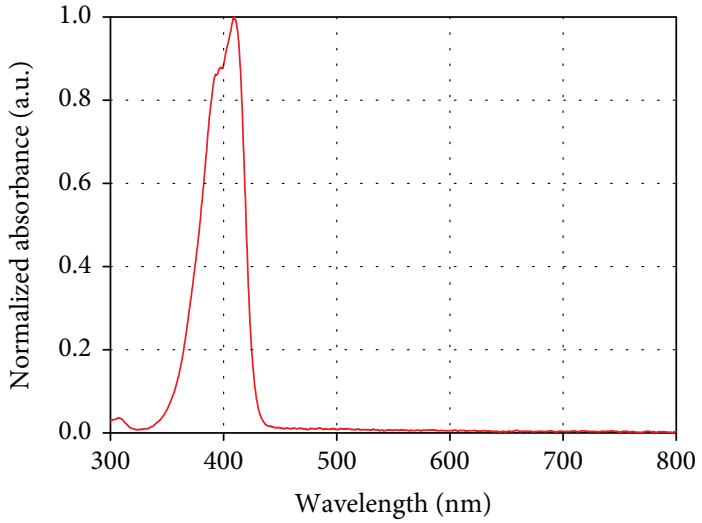

(b)

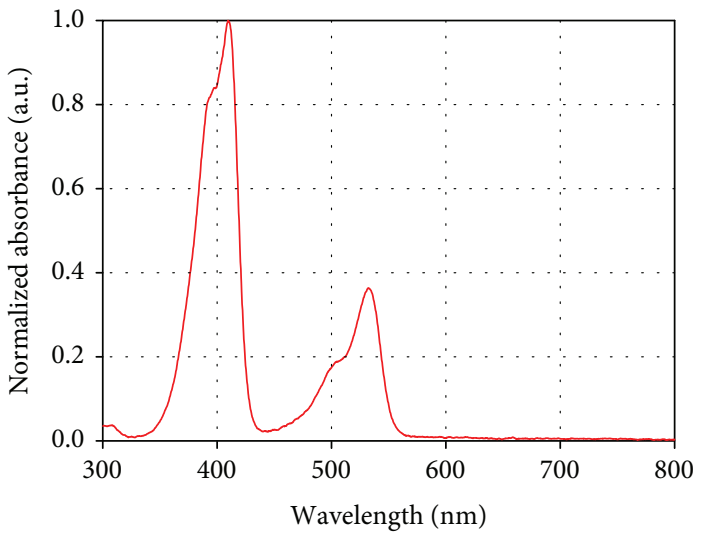

(d)

Figure 6: (a) Photograph of home-made phantom mimicking the blood vessel in the superficial layer under the illumination with white light. (b-d) The absorption spectrums of Dye solution 1, Dye solution 2, and Dye solution 3, respectively.

light is useful for obtaining molecular information such as concentration and structure [25].

Changes in the emission spectrum of the QD film were observed during the continuous illumination. In the experiment, the emission spectrum of the $100 \mathrm{~mm}$ thick film with green QD was measured under the illumination of a UV
LED operating at $40 \mathrm{~mA}$ forward current (about $120 \mathrm{~mW}$ was applied to the LED). Figure 5(a) shows the change in peak position in the 410 and $540 \mathrm{~nm}$ bands over time. It has been observed that the peak position has remained almost unchanged at a glance. The standard deviation of the change in peak position was 0.12 and $0.33 \mathrm{~nm}$ in the 


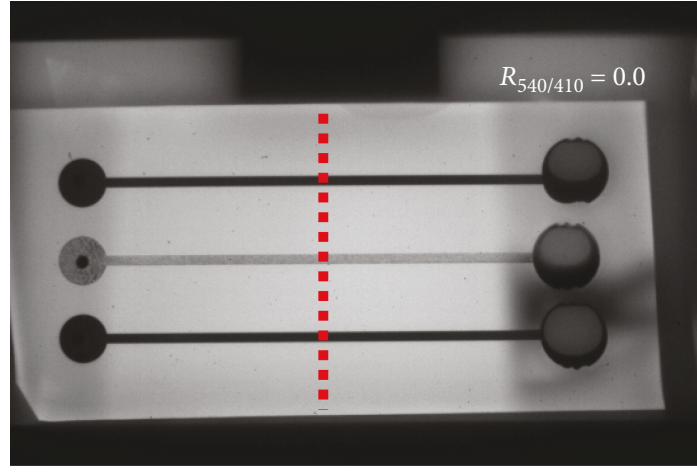

(a)

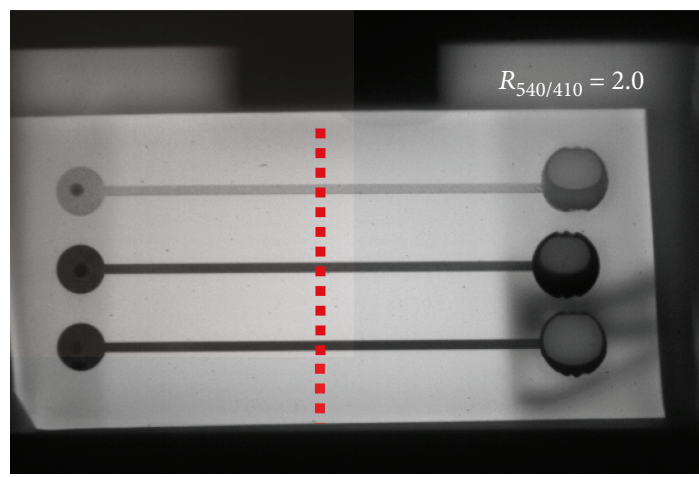

(c)

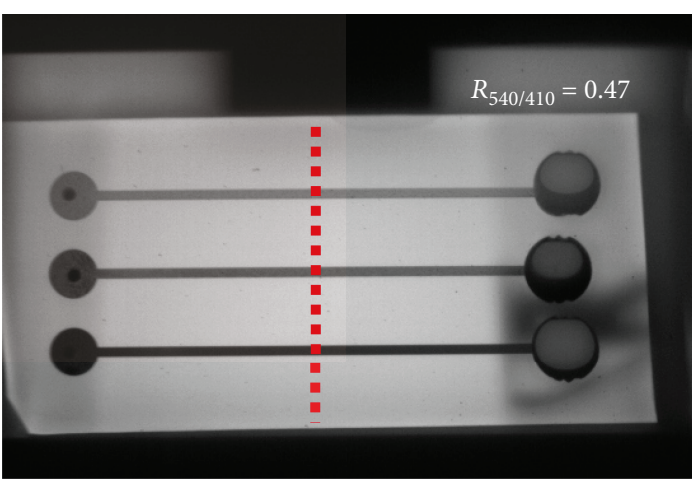

(b)

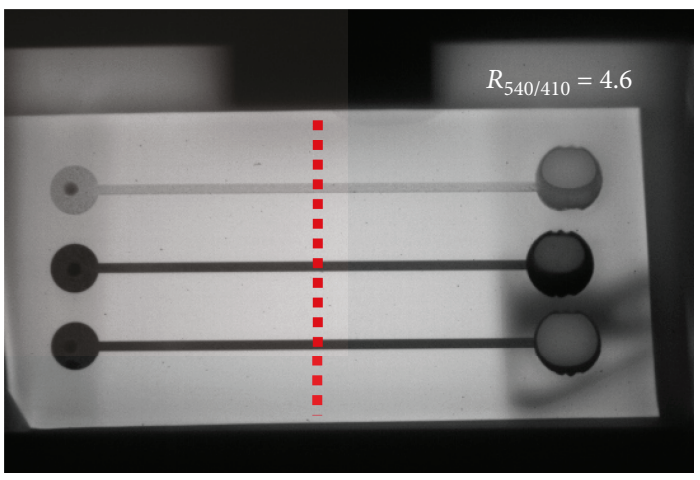

(d)

Figure 7: Photographs of the phantom under various illumination in which the ratio of maximal peak near $540 \mathrm{~nm}$ wavelength to maximal peak near $410 \mathrm{~nm}$ wavelength $\left(R_{540 / 410}\right)$ increases.

410 and $540 \mathrm{~nm}$ bands, respectively. In addition, there was no significant change in $R_{540 / 410}$ value as in the change of the peak position as shown in Figure 5(b). The photoluminescence and electroluminescence of QD were known to be stable even at the temperature higher than $80^{\circ} \mathrm{C}[26,27]$. Therefore, if the optical intensity of excitation light is managed adequately, the emission properties of the QD film may be kept to be stable over a long time.

The effect of QD film on imaging under the illumination with LED was investigated with a home-made phantom. The blood vessel-mimicking phantom was prepared as shown in Figure 6(a), which was the white light image taken under a white light LED. Microchannel patterns which were $1.0 \mathrm{~mm}$ in width and $0.2 \mathrm{~mm}$ in depth were engraved on PDMS and covered with a glass plate which was coated with a scattering medium. The scattering medium was the mixture of PDMS and alumina particle with a diameter of $300 \mathrm{~nm}$, and the thickness and the scattering coefficient were estimated to be about $200 \mu \mathrm{m}$ and $100 / \mathrm{cm}$, respectively. Each channel of the phantom was filled with a dye solution (QCR Solutions Corp., USA) having the absorption spectrum shown in Figures 6(b)-6(d). The absorption peaks of Dye solution 1 and Dye solution 2 are $409.3 \mathrm{~nm}$ and $534.6 \mathrm{~nm}$, respectively. As described above, the absorption peak wavelength almost coincides with the absorption peak wavelength of hemoglobin [19]. Dye solution 3, which is a mixture of Dye solution 1 and Dye solution 2 in a weight ratio of $1: 0.13$, exhibits absorption peaks at both $409.3 \mathrm{~nm}$ and $534.6 \mathrm{~nm}$. Since the light intensity near $410 \mathrm{~nm}$ band is negligible in the white light LED, the pattern of Dye solution 1 compared to the patterns of Dye solution 2 and Dye solution 3 is ambiguous in Figure 6(a).

The phantom images were acquired under various illuminations as shown in Figure 7. No image processing algorithm was applied to all images. When compared to Figure 6(a) obtained under white light illumination, the pattern of Dye solution 1 clearly appears but becomes increasingly fainter as the $R_{540 / 410}$ value increases. On the contrary, the pattern of Dye solution 2 became more vivid as the $R_{540 / 410}$ value increased. The changes in these patterns were quantified as Weber contrast, as shown in Figure 8 [28]. The contrast of the pattern of Dye solution 1 under the illumination of only the spectral band around $410 \mathrm{~nm}$ $\left(R_{540 / 410}=0\right)$ was four times higher than that under the illumination of the white light. This is because the wavelength at the maximum absorption peak of Dye solution 1 lies in the spectral bandwidth of the illumination. Similarly, the contrast of the pattern of Dye solution 2 rose as the $R_{540 / 410}$ value increased. On the other hand, the contrast of pattern of Dye solution 3 decreased slightly with increasing $R_{540 / 410}$. This is because Dye solution 3 has the absorption peaks in both spectral bands.

As mentioned above, the two spectral bands of $410 \mathrm{~nm}$ and $540 \mathrm{~nm}$ correspond to the absorption peak of hemoglobin in common. In the visible range, the light of long wavelength penetrates into deeper areas $[10,12,23]$. Thus, in spectral imaging such as NBI, the blood vessels of superficial mucosa are imaged under illumination of the spectral band 


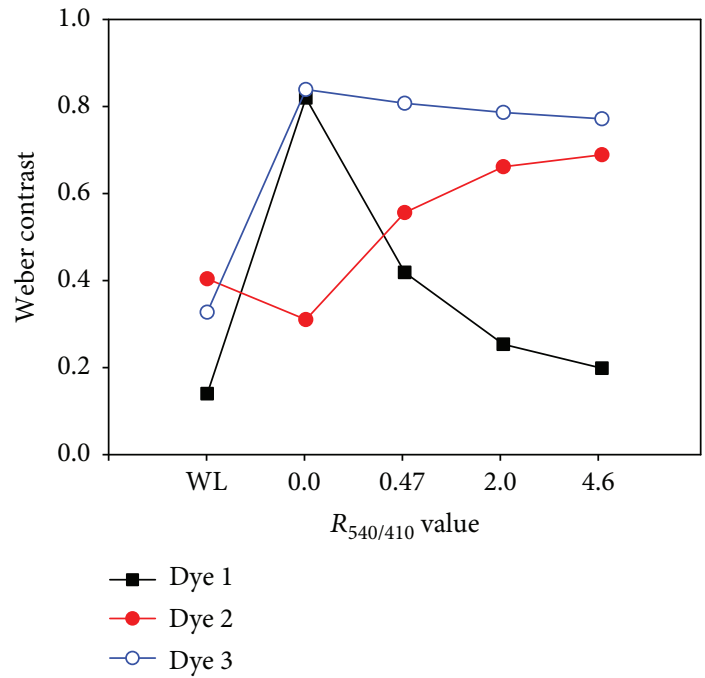

FIGURE 8: Weber contrast of each dye solution under the illuminations of white light (WL) and the light of the various spectral bands.

around $410 \mathrm{~nm}$, and the blood vessels of the deep region or submucosa are imaged under illumination of the spectral band around $540 \mathrm{~nm}$. That is, the illumination of the multispectral bands near 410 and $540 \mathrm{~nm}$ is required to keep the contrast of the patterns of both surface and submucosal blood vessels uniform. In the phantom studies above, the pattern of Dye solution 1 may correspond to the blood vessels of the surface layer, and the pattern of Dye solution 2 could be considered as the vessels of deep tissue. Thus, the LED light source combined with QD films provides a new solution for generating multispectral band light in spectral imaging.

\section{Conclusion}

We showed that LED combined with the quantum dot (QD) film can be used as the light source with multiple spectral bands for spectral imaging. When irradiated with LED, the light with the multiple bands near 410 and $540 \mathrm{~nm}$ was emitted from the QD film, which was the polymer film with semitransparency. The ratio between the intensities at the peak wavelength of both bands can be controlled by the film thickness. LED combined with the QD film can simplify the optical configuration for the illumination of the multispectral bands. In the study using a phantom mimicking blood vessels, it was confirmed that the image of the blood vessel pattern could be improved by LED combined with QD as compared with the white light image. Therefore, the LED light sources combined with QD films provide a new solution for generating multispectral band light.

\section{Data Availability}

The data used to support the findings of this study are included within the article.

\section{Conflicts of Interest}

The authors declare that there is no conflict of interests regarding the publication of this paper.

\section{Authors' Contributions}

Young Min Bae and Dong-Goo Kang contributed equally to this work.

\section{Acknowledgments}

This research was supported by the Korea Electrotechnology Research Institute (KERI) Primary research program through the National Research Council of Science \& Technology (NST) funded by the Ministry of Science and ICT (MSIT) (19-12-N0101-90 and 17-12-N0103-10).

\section{References}

[1] Y. Shiraski, G. J. Supran, M. G. Bawendi, and V. Bulovic, "Emergence of colloidal quantum-dot light-emitting technologies," Nature Photonics, vol. 7, no. 1, pp. 13-23, 2013.

[2] T.-H. Kim, K. S. Cho, E. K. Lee et al., "Full-colour quantum dot displays fabricated by transfer printing," Nature Photonics, vol. 5, no. 3, pp. 176-182, 2011.

[3] W. K. Bae, J. Kwak, J. Lim et al., "Multicolored light-emitting diodes based on all-quantum-dot multilayer films using layer-by-layer assembly method," Nano Letters, vol. 10, no. 7, pp. 2368-2373, 2010.

[4] S. Mazumder, R. Dey, M. K. Mitra, S. Mukherjee, and G. C. Das, "Review: biofunctionalized quantum dots in biology and medicine," Journal of Nanomaterials, vol. 2009, Article ID 815734, 17 pages, 2009.

[5] I. L. Medintz, H. T. Uyesa, E. R. Goldman, and H. Mattoussi, "Quantum dot bioconjugates for imaging, labelling and sensing," Nature Materials, vol. 4, no. 6, pp. 435-446, 2005.

[6] ASGE technology Committee, R. S. Kwon, D. G. Adler et al., "High-resolution and high-magnification endoscopes," Gastrointestinal Endoscopy, vol. 69, no. 3, pp. 399-407, 2009.

[7] J. Y. Jang, "The past, present, and future of image-enhanced endoscopy," Clinical endoscopy, vol. 48, no. 6, pp. 466-475, 2015.

[8] J. H. Lee and T. Dwang, "Molecular endoscopy for targeted imaging in the digestive tract," The Lancet Gastroenterology \& Hepatology, vol. 1, no. 2, pp. 147-155, 2016.

[9] J. Burggraaf, I. M. C. Kamerling, P. B. Gordon et al., "Detection of colorectal polyps in humans using an intravenously administered fluorescent peptide targeted against c-Met," Nature Medicine, vol. 21, no. 8, pp. 955-961, 2015.

[10] ASGE technology Committee, L. M. W. K. Song, D. G. Adler et al., "Narrow band imaging and multiband imaging," Gastrointestinal Endoscopy, vol. 67, no. 4, pp. 581-589, 2008.

[11] K. Togashi, D. Nemoto, K. Utano et al., "Blue laser imaging endoscopy system for the early detection and characterization of colorectal lesions: a guide for the endoscopist," Therapeutic Advances in Gastroenterology, vol. 9, no. 1, pp. 50-56, 2016.

[12] K. Gono, "Narrow band imaging: technology basis and research and development history," Clinical Endoscopy, vol. 48, no. 6, pp. 476-480, 2015. 
[13] V. Ntziachristos, J. Ripoll, L. V. Wang, and R. Weissleder, "Looking and listening to light: the evolution of whole-body photonic imaging," Nature Biotechnology, vol. 23, no. 3, pp. 313-320, 2005.

[14] B. P. Joshi, X. Duan, R. S. Kwon et al., "Multimodal endoscope can quantify wide-field fluorescence detection of Barrett's neoplasia," Endoscopy, vol. 48, no. 2, pp. A1-A13, 2016.

[15] H.-J. Chang, W.-H. Wang, Y.-L. Chang et al., "Light-emitting diode-assisted narrow band imaging video endoscopy system in head and neck cancer," Clinical Endoscopy, vol. 48, no. 2, pp. 142-146, 2015.

[16] S. Muthu, F. J. P. Schuuramans, and M. D. Pashley, "Red, green, and blue LED for white light illumination," IEEE Journal on Selected Topics in Quantum Electronics, vol. 8, no. 2, pp. 333-338, 2002.

[17] J. I. Kim and J.-K. Lee, "Sub-kilogram-scale one-pot synthesis of highly luminescent and monodisperse core/shell quantum dots by the successive injection of precursors," Advanced Materials, vol. 16, no. 16, pp. 2077-2082, 2006.

[18] Y. M. Bae, K.-H. Lee, J. Yang, D. Heo, and H. J. Cho, "Hydrogel-based capillary flow pumping in a hydrophobic microfluidic channel," Japanese Journal of Applied Physics, vol. 53, no. 6, article 067201, 2014.

[19] M. Nitzan, A. Romem, and R. Koppel, "Pulse oximetry: fundamentals and technology update," Medical devices: Evidence and Research, vol. 7, p. 231, 2014.

[20] E. Moon, M. Kim, J. Roh, H. Kim, and J. Hahn, "Holographic head-mounted display with RGB light emitting diode light source," Optics Express, vol. 22, no. 6, pp. 6526-6534, 2014.

[21] Y. Garini and E. Tauber, "Spectral imaging: methods, design, and applications," in Biomedical optical imaging technologies, Biological and Medical Physics, Biomedical Engineering, R. Liang, Ed., pp. 111-161, Springer, Berlin, Heidelberg, 2012.

[22] M. Bruchez, M. Moronne, P. Gin, S. Weiss, and A. P. Alivisatos, "Semiconductor nanocrystals as fluorescent biological labels," Science, vol. 281, no. 5385, pp. 2013-2016, 1998.

[23] M. Muto, C. Katada, Y. Sano, and S. Yoshida, "Narrow band imaging: a new diagnostic approach to visualize angiogenesis in superficial neoplasia," Clinical Gastroenterology and Hepatology, vol. 3, no. 7, Supplement 1, pp. S16-S20, 2005.

[24] M. N. Berberan-Santos, "Beer's law revisited," Journal of Chemical Education, vol. 67, no. 9, pp. 757-759, 1990.

[25] X. Yu, Q. Lu, H. Gao, and H. Ding, "Development of a handheld-spectrometer based on a linear variable filter and a complementary metal-oxide semiconductor detector for measuring the internal quality of fruit," Journal of Near Infrared Spectroscopy, vol. 24, no. 1, pp. 69-76, 2016.

[26] Y. Fu, D. Kim, W. Jiang, W. Yin, T. K. Ahn, and H. Chae, "Excellent stability of thicker shell CdSe@ZnS/ZnS quantum dots," RSC Advances, vol. 7, no. 65, pp. 40866-40872, 2017.

[27] H.-T. Vu, R.-K. Chiang, C.-Y. Huang et al., "Enhanced thermal stability of green-emission quantum-dot light-emitting diodes via composition-gradient thick-shell quantum dots," Applied Physics Express, vol. 9, no. 8, article 082101, 2016.

[28] E. Peli, "Contrast in complex images," Journal of Optical Society America A, vol. 7, no. 10, pp. 2032-2040, 1990. 


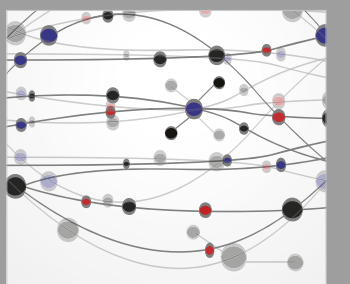

The Scientific World Journal
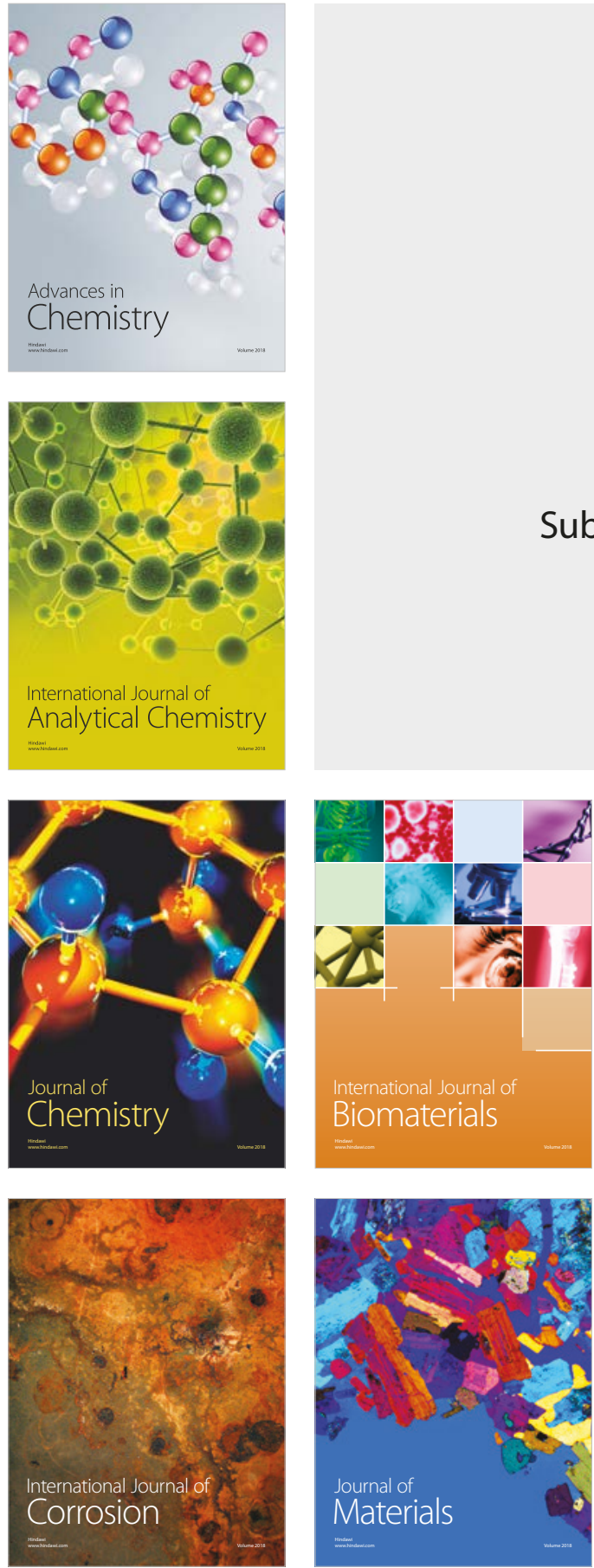

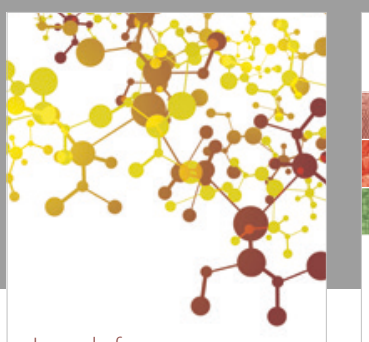

Journal of

Applied Chemistry
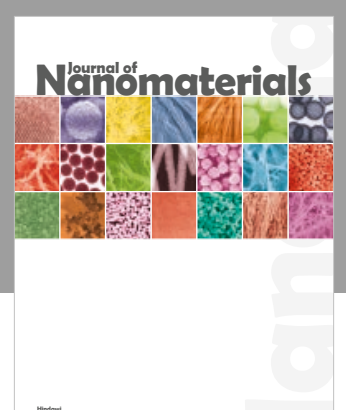

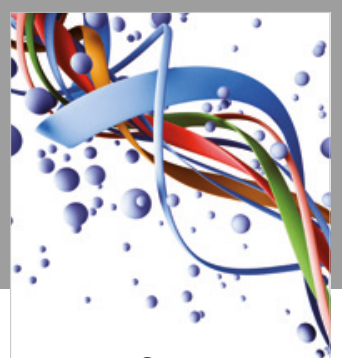

Scientifica

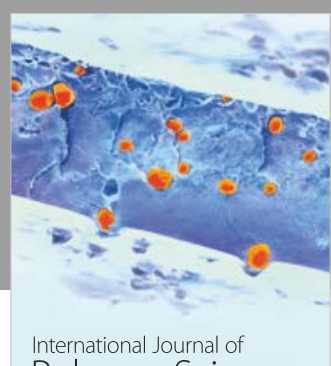

Polymer Science

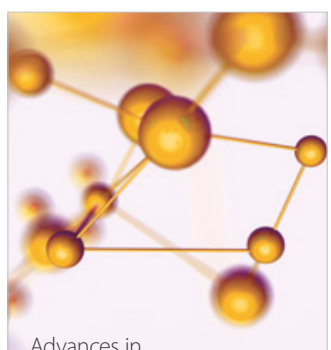

Physical Chemistry
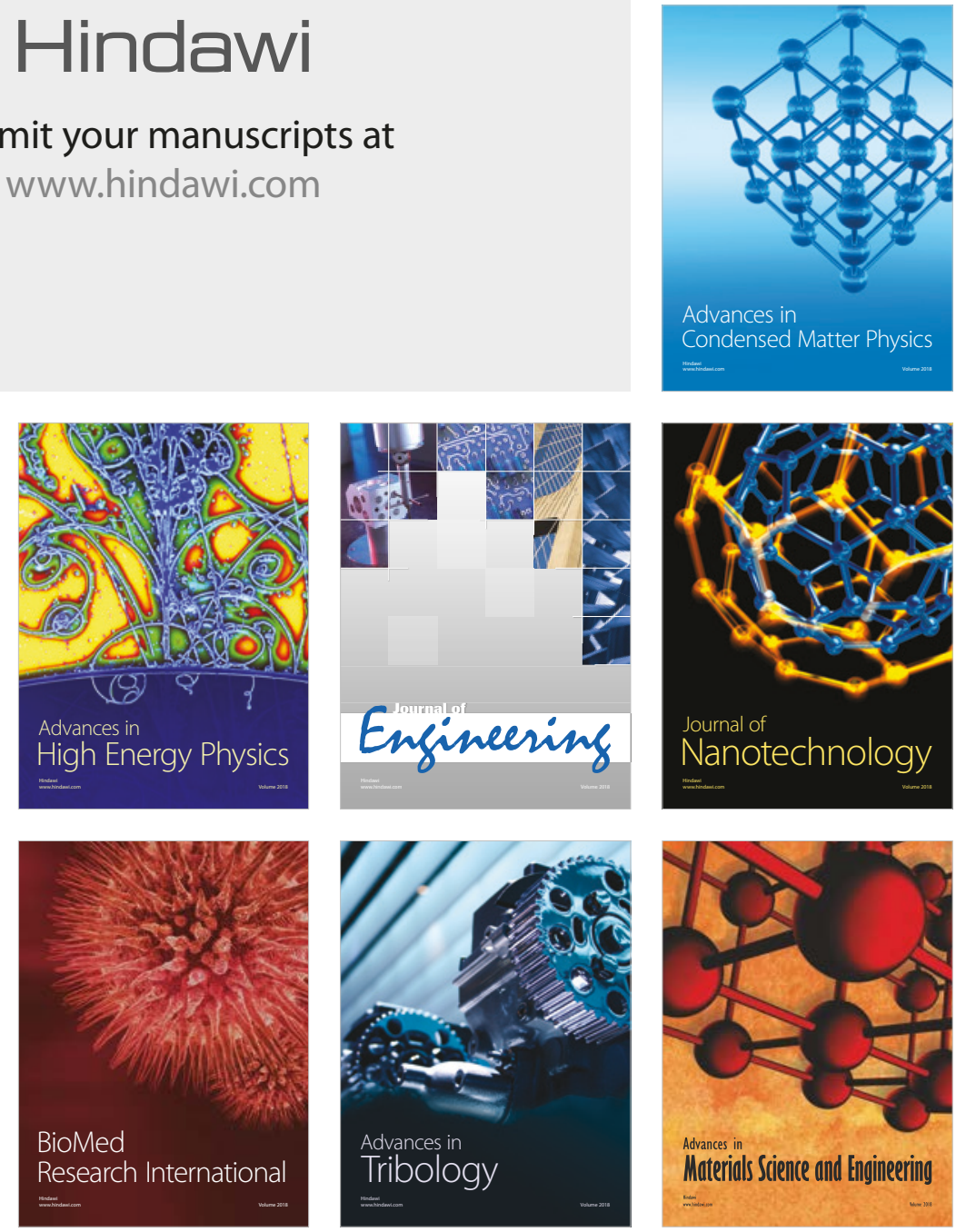